\title{
40. ICHTHYOLITHS IN SOME SAMPLES FROM THE PHILIPPINE SEA, DEEP SEA DRILLING PROJECT LEG 31
}

\author{
A. T. Dengler, P. S. Doyle, and W. R. Riedel, Scripps Institution of Oceanography, La Jolla, California
}

\section{MATERIAL EXAMINED}

The scientific party aboard D/V Glomar Challenger for Leg 31 took a number of samples for analysis of fish skeletal debris in otherwise unfossiliferous sequences. In this paper we briefly report the results of examination of ichthyoliths from the following drilling sites:

Site 293: $20^{\circ} 21.25^{\prime} \mathrm{N}, 124^{\circ} 05.65^{\prime} \mathrm{E}$, water depth 5599 meters

Site $294: 22^{\circ} 34.74^{\prime} \mathrm{N}, 131^{\circ} 23.13^{\prime} \mathrm{E}$, water depth 5784 meters

Site $295: 22^{\circ} 33.76^{\prime} \mathrm{N}, 131^{\circ} 22.04^{\prime} \mathrm{E}$, water depth 5802 meters

Site $297: 30^{\circ} 52.36^{\prime} \mathrm{N}, 134^{\circ} 09.89^{\prime} \mathrm{E}$, water depth 4458 meters

The system of discrimination and nomenclature used is that developed by Doyle et al. (1974) for material collected on DSDP Leg 26.

\section{RESULTS}

Table 1 shows the numbers of specimens found, of stratigraphically significant forms, and their time ranges as determined in the Leg 26 Initial Report. The small number of specimens recorded, for many of the "taxa," reflects the rather small size of the samples submitted-it is generally desirable to have sediment samples of at least $30-50 \mathrm{cc}$, and these can usually be easily spared from otherwise unfossiliferous cores.

\section{Hole 293}

On the basis of other microfossil groups, the material in Core 9 (above the interval that we have examined) is late Pliocene, and that below (Core 20) is late middle Miocene. As shown in Table 1, the ichthyoliths indicate that Cores 12-15 are between late Miocene and late Pliocene in age, most probably late Miocene.

\section{Hole 294}

The ichthyolith assemblage in Core 1, Section 2, indicates a late Miocene age; those in Core 1, Section 4, and Core 2 a late Oligocene to early Miocene age; that in Core 3 an early Oligocene age or older; and that in Core 4 (putting together the results from Sections 2, 4, and 6) indicates an early Oligocene age, except for the occurrence of one form (Kite-shaped irregular network) represented in the Leg 26 Initial Report by two specimens in early Eocene assemblages. The assemblage in Core 6 includes two hitherto undescribed forms (Plate 5, Figures 5, 6) not found before in Oligocene and younger deposits, and two known subtypes from sediments at least as young as early Eocene and thus may be Eocene or older.

\section{Hole 295}

The assemblage in Core 1, taking Sections 3 and 5 together, indicates an age of late Oligocene to early Miocene. That in Core 2, taking Sections 2 and 6 together, indicates a late Eocene to early Oligocene age, but this depends on a form (Five peaks flared base) of which only three specimens have been found in assemblages of known age. The ichthyoliths in Cores 3 and $3 \mathrm{~A}$ indicate only that the sediments are late Oligocene or older.

\section{Hole 297}

Too few identifiable ichthyoliths were found in eight samples from Cores 10 through 26 to permit an age assignment.

\section{ACKNOWLEDGMENTS}

This research was supported partly by NSF Grant GA40158 , and partly by the University of California. Jean M. Westberg and Grace G. Kennedy assisted in making the microscopic preparations, and Marguerite J. Dunsworth contributed useful discussion. M. A. Hanger and M. A. Neely typed and proofread the manuscript.

\section{REFERENCE}

Doyle, P. S., Kennedy, G. G., and Riedel, W. R., 1974. Stratignathy. In Davies, T. A., Luyendyk, B. P., et al., Initial Reports of the Deep Sea Drilling Project, Volume 26: Washington (U.S. Government Printing Office), p. 825906.

\section{PLATES}

Here are illustrated all of the identified ichthyoliths recorded in this paper, as well as several notable forms not yet described. Their arrangement follows the system of name-descriptors developed in the Initial Report for Leg 26. In the explanations to the figures, the sample numbers and slide designations (in the form "S1.4", etc.) indicate preparations in our collection at Scripps Institution of Oceanography, and designations in the form "R 45/1" indicate England Finder positions of the illustrated specimens on the slides.

The authorship and date which is an essential part of each name-description, is identical (Doyle et al., in press) for all forms recorded herein, and is therefore omitted here, though this will not be possible in later papers when the descriptive scheme becomes modified and the stratignathic literature more voluminous. 
TABLE

Distribution of Icthyolith Subtypes at Sites 293, 294, and 295

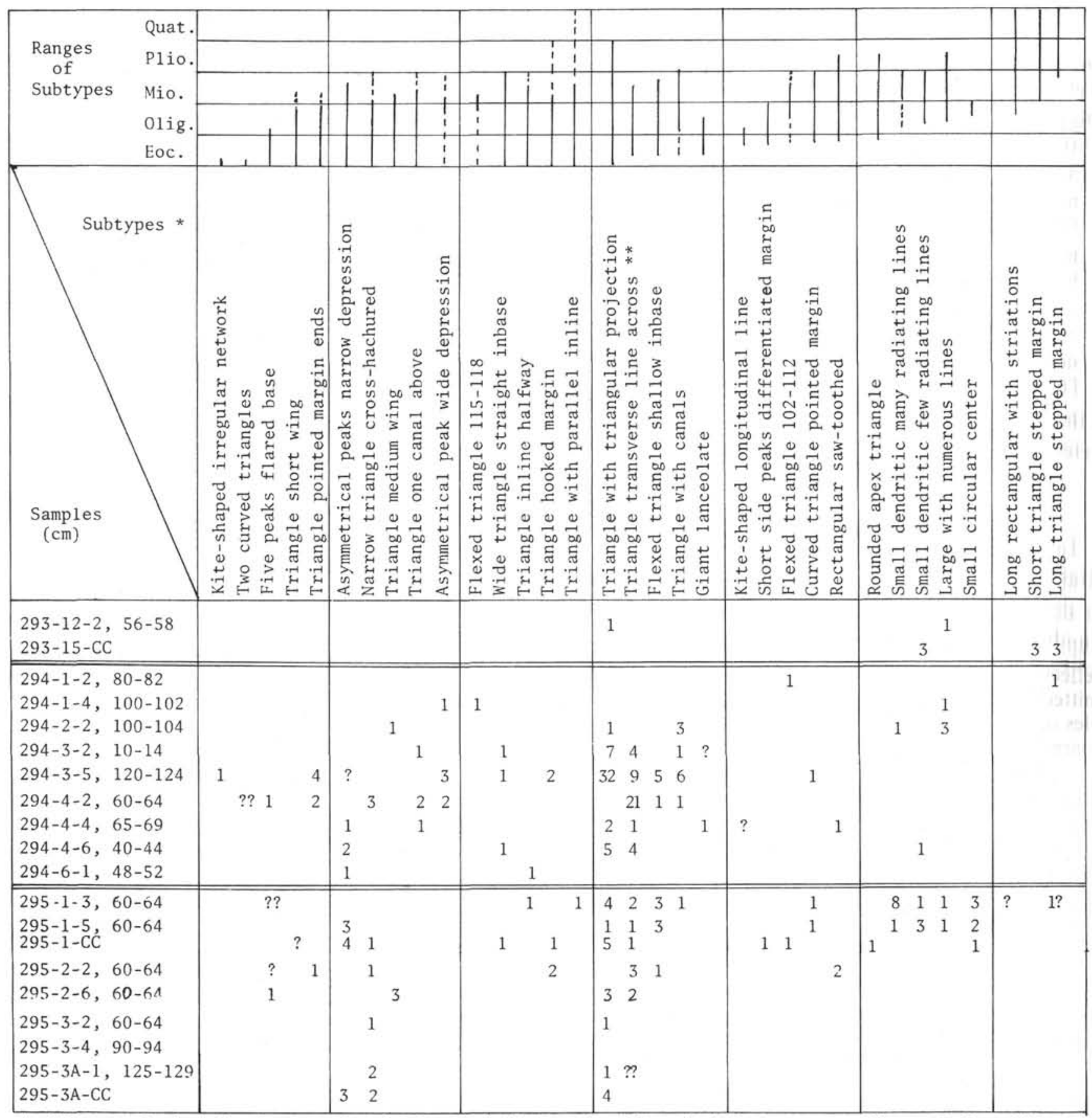

* Subtypes looked for but not found: Three similar peaks; Skewed with transverse lines; Tall median peak transverse lines; Three equal peaks flared base; Skewed four or five peaks; Short kite-shaped; Kite-shaped elongate prominence; Rhombus smooth margin; Plain lanceolate; Circular with line across; Elliptical with line across; Triangle crenulate; Short rectangular with constrictions; Triangle crenulate with canals; Triangle saw-toothed margin.

** Tabulations for this subtype include the undescribed form pictured in Plate 3, Figure 13. 



\section{PLATE 1}

(Magnification 110X)

Figure 1

Figure 2

Figure 3

Figure 4

Figure 5

Figure 6

Figure 7

Figures 8-10 $a 2 / b 2 / c 3 / d l / e l / f 3 / g l / h 3 / i l / j l, 2$; Short side peaks differentiated margin.

295-1, CC, S1.3, R32/2.

$a 2 / b 2 / c 5 / d 3 / e l / f l / g l / h 4 / i l ~ / ~ j l, 2$;

Five peaks flared base.

294-4-2, 60-64 cm, S1.2, M28/1.

$a 3 / b 1 / c 3 / d 4 / e 2 / f 2 / g l+4$;

Kite-shaped irregular network.

294-3-5, 120-124 cm, S1.4, E11/2.

$a 4 / b l / c l / d 3 / e 0 / f 2+3 / g l+2$;

Giant lanceolate.

294-3-5, 120-124 cm, S1.1, T39/2.

$a 5,6 / b 3 / c l$ / dl / e2;

Small dendritic few radiating lines.

295-1-3, 60-64 cm, S1.2, U26/3.

$a 5,6 / b 3 / c l / d 2 / e 2$;

Small dendritic many radiating lines.

295-1-3, 60-64 cm, S1.1, R29/0.

$a 5,6$ / b3 / c2 / dl,2 / el;

Large with numerous lines.

295-1-3, 60-64 cm, S1.1, Q42/3.

$a 5,6 / b 3 / c l / d 3 / e 3$;

Small circular center.

8. 295-1-5, 60-64 cm, S1.1, H13/0.

9. 295-1, CC, S1.1, W32/1.

10. 295-1-3, 60-64 cm, S1.2, O14/0.

Figures 11, $12 a 7 / b 1 / c 2 / d 3 / e 3$;

Rectangular saw-toothed.

11. 294-4-4, 65-69 cm, S1.1, R27/0.

12. $295-2-2,60-64 \mathrm{~cm}, \mathrm{~S} 1.1, \mathrm{O} 20 / 0$.

Figure $13 \quad a 7 / b 6 / c 3$;

Asymmetrical peaks narrow depression. 294-6-1, 48-52 cm, S1.2, F18/1.

Figures 14, $15 \quad a 7 / b 6 / c l$;

Asymmetrical peak wide depression.

14. 294-4-2, 60-64 cm, S1.2, T12/1.

15. 294-3-5, 120-124 cm, S1.1, E10/3. 
PLATE 1
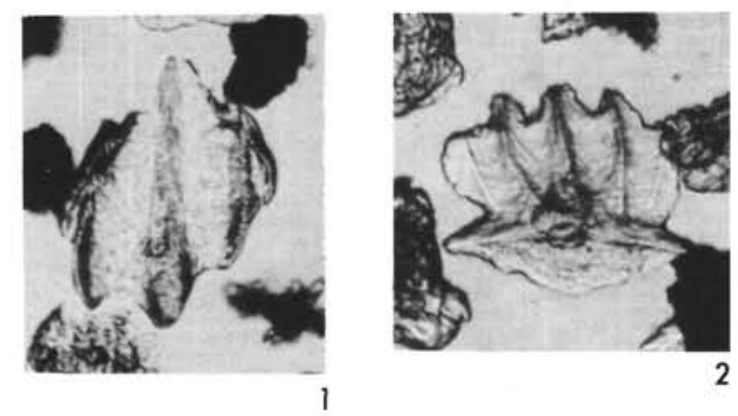
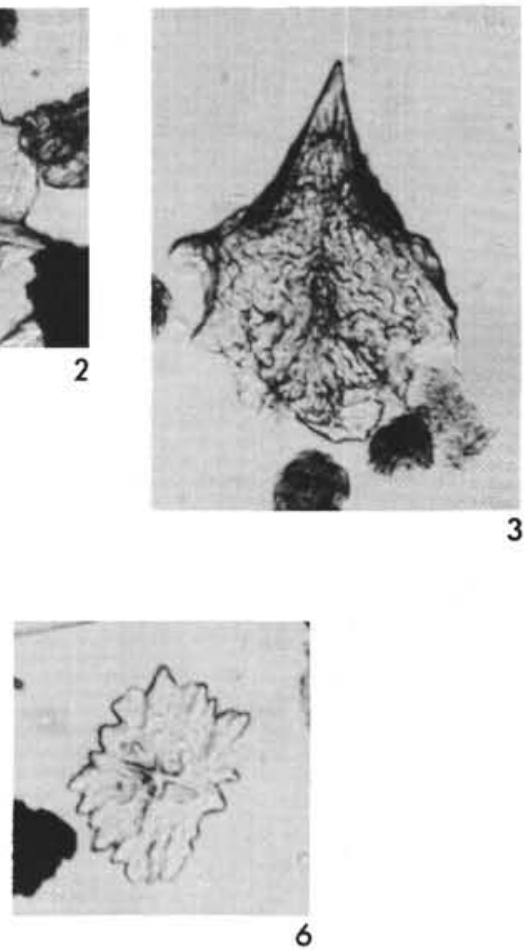
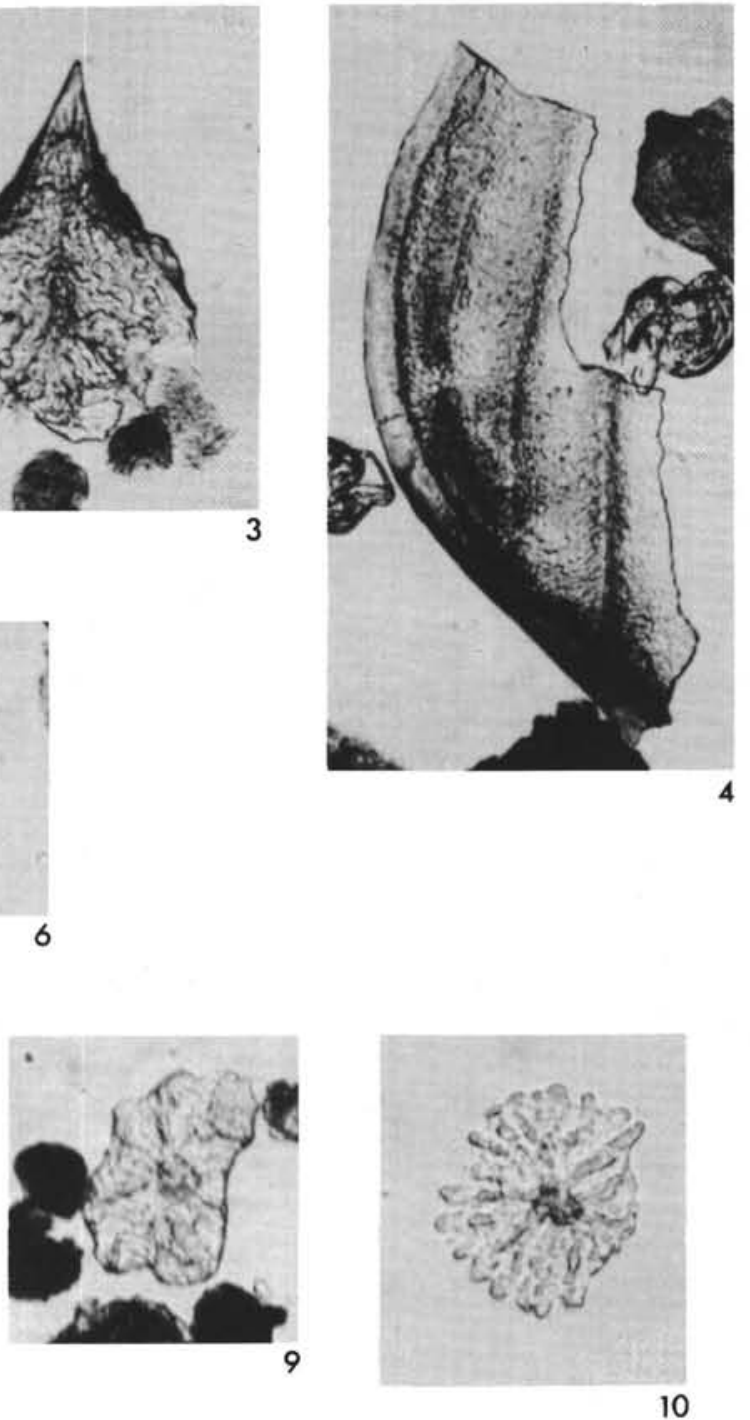

10

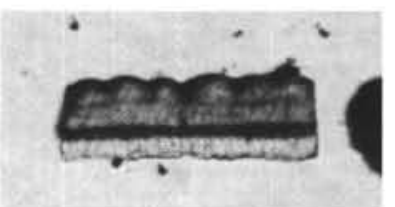

11

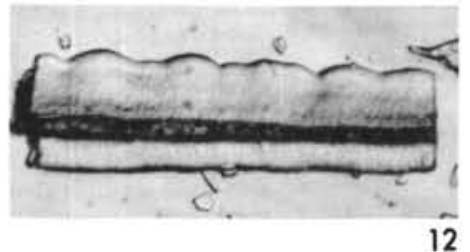

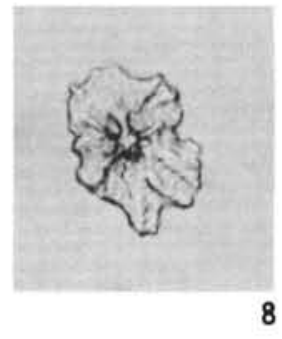
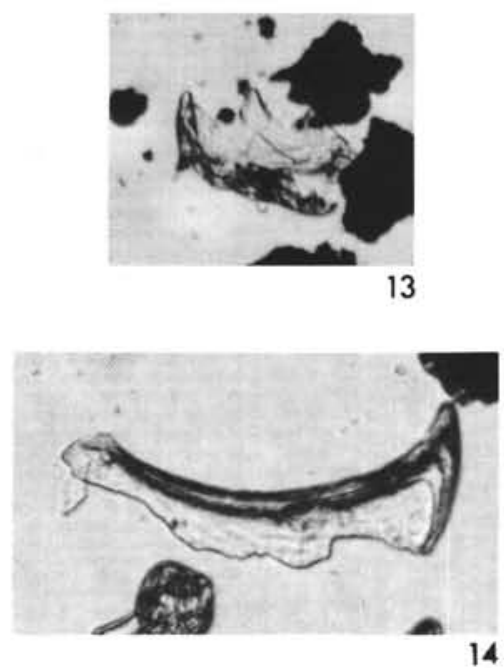
PLATE 2

(Magnification $110 \times$ )

Figures 1,2 as / bl / cl / dl / el02-l12 / f26-36;

Flexed triangle 102-112.

1. 294-1-2, 80-82 cm, S1.2, J29/3.

2. 295-1, CC, S1.3, M25/0.

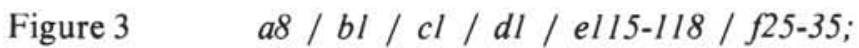

Flexed triangle 115-118.

294-1-4, 100-102 cm, S1.5, Q24/1.

Figure $4 \quad a 8 / b l / c l / d 2 / e 80-140 / f 26-36$;

Flexed triangle shallow inbase.

294-3-2, 10-14 cm, S1.6, J20/2.

Figure $5 \quad a 9 / b l / c l / d l / e l / f l / g l / h l, 2,3 / i 2,3 / j 2,3$ / k2 / 10.25-0.45 / ml-1.5 / n4,5 / ol / p3;

Wide triangle straight inbase.

294-3-2, 10-14 cm, S1.4, Q22/0.

Figure $6 \quad a 9 / b l / c l / d l / e l / f 2 / g l / h l, 2,3 / i 6 / j 4$ / $k 2,4$ / lo.2-0.4 / ml-2 / n3,4,5 / o2 / pl;

Curved triangle pointed margin.

295-1-3, 60-64 cm, S1.2, D38/1.

Figure 7

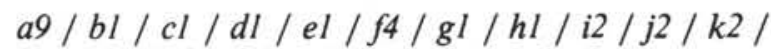
10.45-0.55 / m2.5-3.5 / n2 / ol,5 / p2;

Triangle inline halfway.

295-1-3, 60-64 cm, S1.1, P20/1.

Figure $8 \quad a 9 / b l / c l / d l / e l / f l, 4 / g l / h l / i 2 / j 2 / k 7 /$ 10.5-0.7 / m2.5-3.5 / n2 / ol / pl,2;

Triangle with parallel inline.

295-1-3, 60-64 cm, S1.1, O12/2.

Figure $9 \quad a 9 / b l / c 5 / d l / e l / f l, 4,5 / g l / h l, 3 / i 2,3 / j 6$ / $\mathrm{k} 2$ / 10 / $\mathrm{mo} / \mathrm{nl} / \mathrm{ol} / \mathrm{pl}$;

Triangle short wing?.

295-1, CC, S1.3, E18/4.

Figure $10 \quad a 9 / b l / c 6,7 / d l / e l / f l, 4,5 / g l / h l, 3 / i 2,3 /$ j6 / $\mathrm{k} 2$ / l<0.4 / $\mathrm{ml} .5-2$ / $\mathrm{nl} / \mathrm{ol} / \mathrm{pl}$;

Triangle medium wing.

295-2-6, 60-64 cm, S1.1, G32/3.

Figure 11 Undescribed form.

294-3-5, 120-124 cm, S1.1, C10/2.

Figure $12 \quad a 9 / b l / c l 3 / d l 3 / e l / f 4 / g l / h l / i 2 / j 2 / k 2 /$

$l<0.4$ / m2.4-3 / n3 / o3 / p2;

Triangle pointed margin ends.

294-3-5, 120-124 cm, S1.1, U31/0. 
PLATE 2
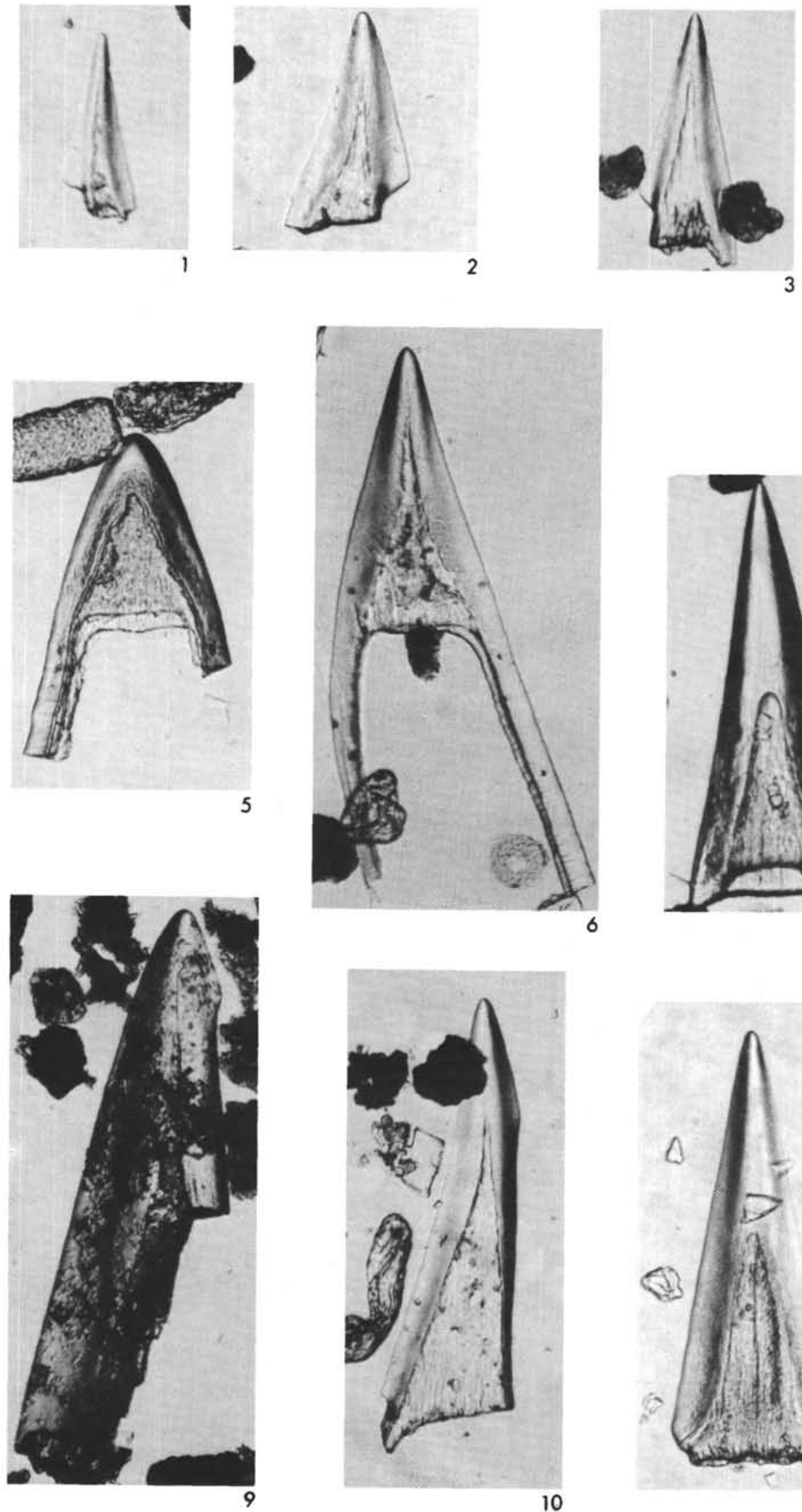
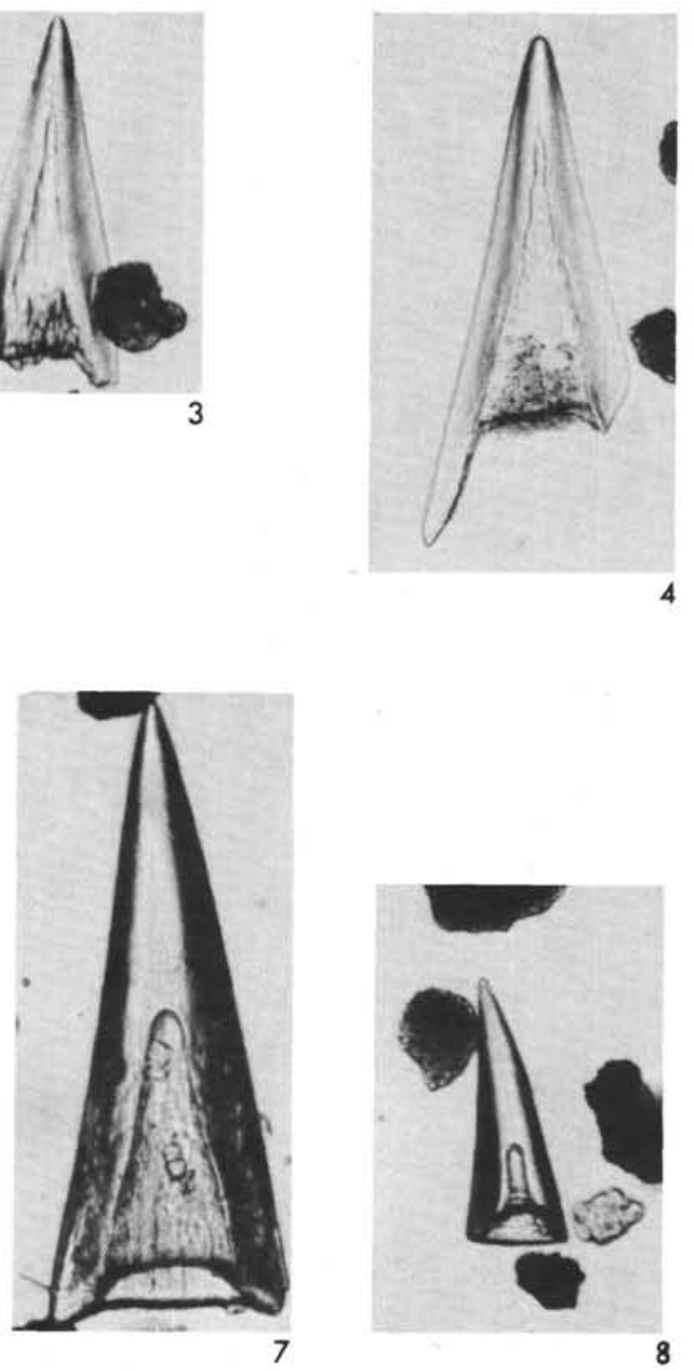
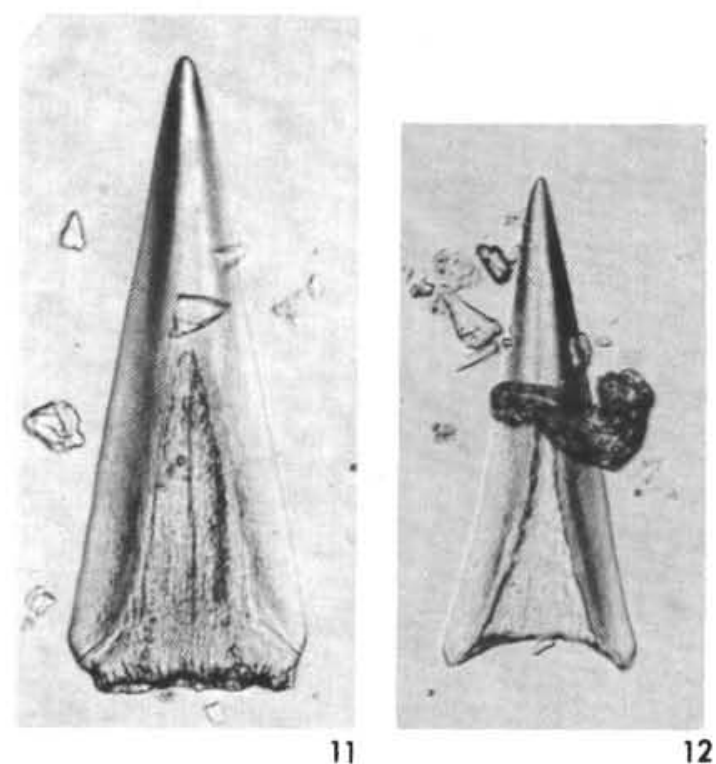
PLATE 3

(Magnification 110×)

Figure $1 \quad a 9 / b l / c l 4 / d l / e l / f 2+5 / g l / h 2 / i 4 / j 2,3 /$ $\mathrm{k} 2 / 10.1-0.4 / \mathrm{ml}-2 / \mathrm{nl} / \mathrm{ol} / \mathrm{pl}+\mathrm{a} 9 / \mathrm{b5} / \mathrm{cl} 3 /$ $\mathrm{dl} / \mathrm{el} / \mathrm{fl} / \mathrm{gl} / \mathrm{hl} / \mathrm{i} 2+7 / \mathrm{jl} / \mathrm{k2} / \mathrm{l4} / \mathrm{m3} / \mathrm{nl}$ / ol / p3 / $q<0.4$ / $r l-2$ / so / $t l$;

Triangle hooked margin.

294-3-5, 120-124 cm, S1.4, U16/3.

Figure $2 \quad a 9 / b 5 / c l / d l / e l / f l / g l / h l / i l, 6 / j 3 ? / k l$ / ll / $m 2 / n 2 / o 3,4 / p 2 / q 0 / r 0 / s>1 / t 2,3$; Long rectangular with striations? 295-1-3, 60-64 cm, S1.1, C11/4.

Figure $3 \quad a 9 / b 5 / \mathrm{cl} / \mathrm{dl} / \mathrm{el} / \mathrm{fl} / \mathrm{gl} / \mathrm{hl} / i l, 3,5 / j l /$ $k 2,6 / l 3 / m 2,4 / n 2,3 / o 3 / p 3,8 / q>0.4 / r<2$ / so / $t 2$;

Short triangle stepped margin.

293-15, CC, S1.1, L33/0.

Figures 4, $5 \quad a 9 / b 5 / \mathrm{cl} / \mathrm{dl} / \mathrm{el} / \mathrm{fl} / \mathrm{gl} / \mathrm{hl} / \mathrm{il}, 5 / \mathrm{jl} / \mathrm{k2,6}$ / $13 / \mathrm{m} 2,4 / \mathrm{n} 2,3 / o 3 / p 3,8 / q>0.4 / r>2 / s 0$ / t2;

Long triangle stepped margin.

4. 293-15, CC, S1.2, C6/3.

5. $294-1-2,80-82 \mathrm{~cm}, \mathrm{~S} 1.1, \mathrm{G} 36 / 4$.

Figure 6

a9 / b5 / cl / dl / e4 / fl / gl / hl / il / jl / k2,7 / $12 / \mathrm{m} 2,4 / \mathrm{n} 3 / \mathrm{ol}, 2 / \mathrm{p} 2,3 / q 0 / r>1 / s>3 / t 2$;

Triangle with triangular projection.

294-3-5, 120-124 cm. S1.1, W15/2.

Figure $7 \quad a 9 / b 5 / c l, 4 / d l / e l, 4 / f l / g l / h l / i 5 / j 2 /$ $k 2,7 / l 2 / m 2,4 / n 2,3 / o l, 2 / p 2,3,6 / q 0 / r>0.5$ / $s>3 / t 2$;

Narrow triangle cross-hachured.

294-4-2, 60-64 cm, S1.2, O30/3.

Figure $8 \quad a 9 / b 5 / \mathrm{cl} / d l / e l / f l / g l, 2 / h 2 / i l, 4 / j l / k 2$ $/ \mathrm{l} / \mathrm{m} 2 / \mathrm{n} 2 / 05,6 / \mathrm{p} 3 / q 0.2-0.5 / \mathrm{rl}-1.5 / \mathrm{s} 0$ / $t l$;

Triangle with canals.

294-3-5, 120-124 cm, S1.1, C10/2.

Figure $9 \quad a 9 / b 5 / c l / d l / e l / f l / g l / h 2 / i 4+(l, 5) / j l /$ $\mathrm{k} 2 / \mathrm{l} 2 / \mathrm{m} 2 / \mathrm{n} 2 / \mathrm{o} 4 \mathrm{p} 8$ / q0.3-0.6 / rl-2 / s0 / $t l$;

Triangle one canal above.

294-3-2, 10-14 cm, S1.2, D42/1.

Figures 10-12 $a 9 / b 5 / c l / d l / e l / f l / g 2 / h 2 / i 4 / j l / k 2,4,6$ / $[2,4 / \mathrm{ml} / \mathrm{nl} / 04 / \mathrm{p3} / \mathrm{q0} .2-0.4$ / rl.5-2.5 / s0 / $t 1$;

Triangle transverse line across.

10. 294-4-2, 60-64 cm, S1.2, S35/0.

11. Same sample, S1.1, H36/3.

12. Same sample, S1.2, E15/0.

Figure 13 Undescribed form. 294-4-2, 60-64 cm, S1.3, O29/4.

Figure $14 \quad a 9 / b 7$; Rounded apex triangle.

295-1, CC, S1.5, D17/4. 
PLATE 3
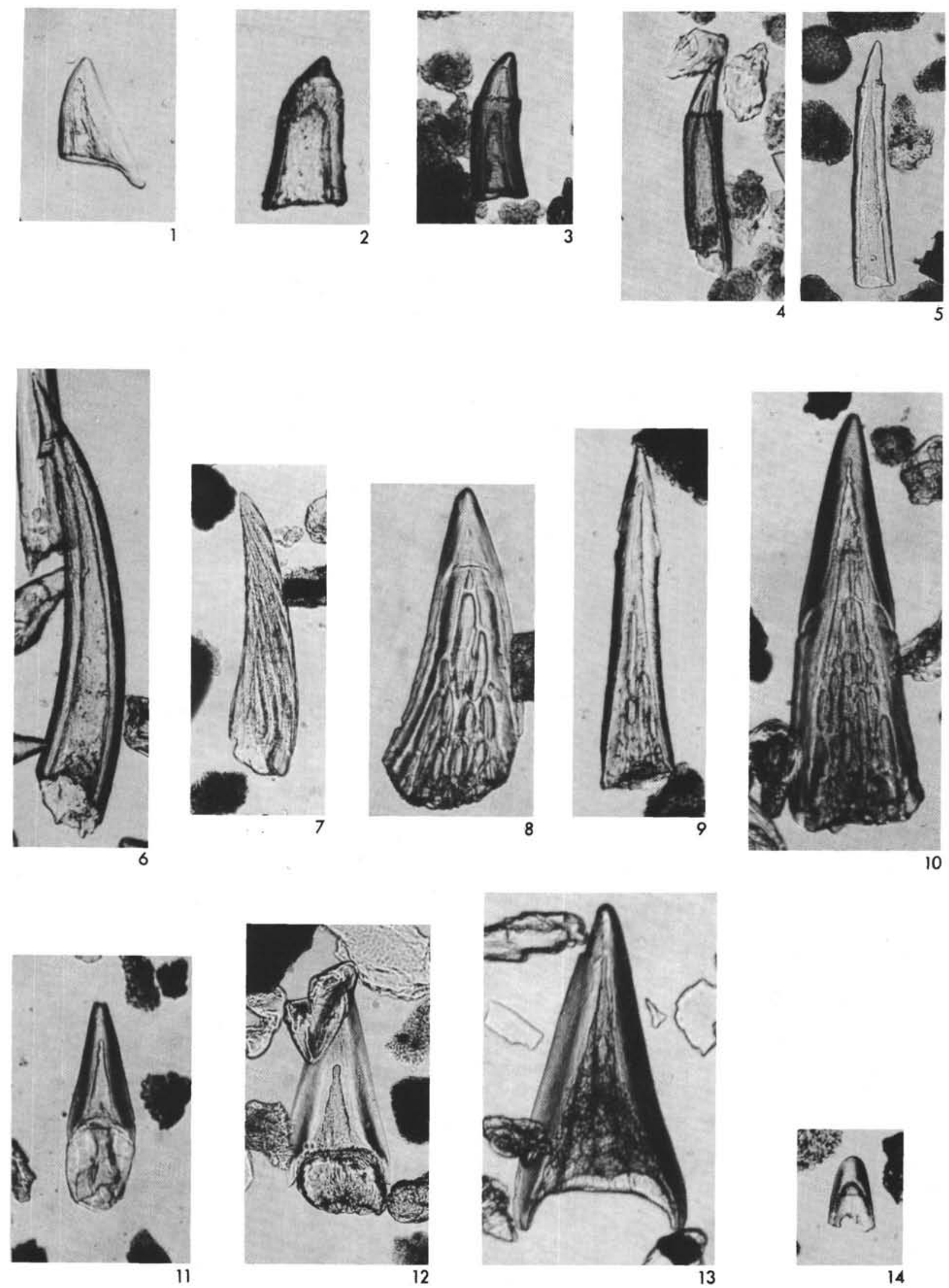


\section{PLATE 4}

(Magnification 110X)

Figures 1-11 Undescribed forms.

1. 294-3-2, 10-14 cm, S1.2, R37/3.

2. 293-15, CC, S1.1, P42/2.

3. 294-4-2, 60-64 cm, S1.2, T26/1.

4. 295-2-2, 60-64 cm, S1.1, P36/4.

5. 294-4-2, 60-64 cm, S1.1, D10/2.

6. Same sample, S1.2, F9/2.

7. 294-6-1, 48-52 cm, S1.4, O24/4.

8. Same sample, S1.2, H24/0.

9. $294-3-2,10-14 \mathrm{~cm}, \mathrm{~S} 1.4, \mathrm{Y} 35 / 0$.

10. 294-2-2, 100-104 cm, S1.3, R46/0.

11. 294-3-2, 10-14 cm, S1.2, X39/3.

Figures 12-16 Undescribed forms (cf. Two curved triangles, Two curved triangles long base, Doyle et al., in press, pl. $2 \mathrm{G}$, fig. 1-3).

12. 295-3A, CC, S1.1, R38/4.

13. $294-4-2,60-64 \mathrm{~cm}, \mathrm{~S} 1.2, \mathrm{~S} 12 / 0$.

14. 294-4-6, 40-44 cm, S1.11, N33/3.

15. 295-3-5, 120-124 cm, S1.3, S33/1.

16. 295-3, CC, S1.1, U39/4.

Figure 17 Undescribed form.

295-3A, CC, S1.1, C18/0. 
PLATE 4
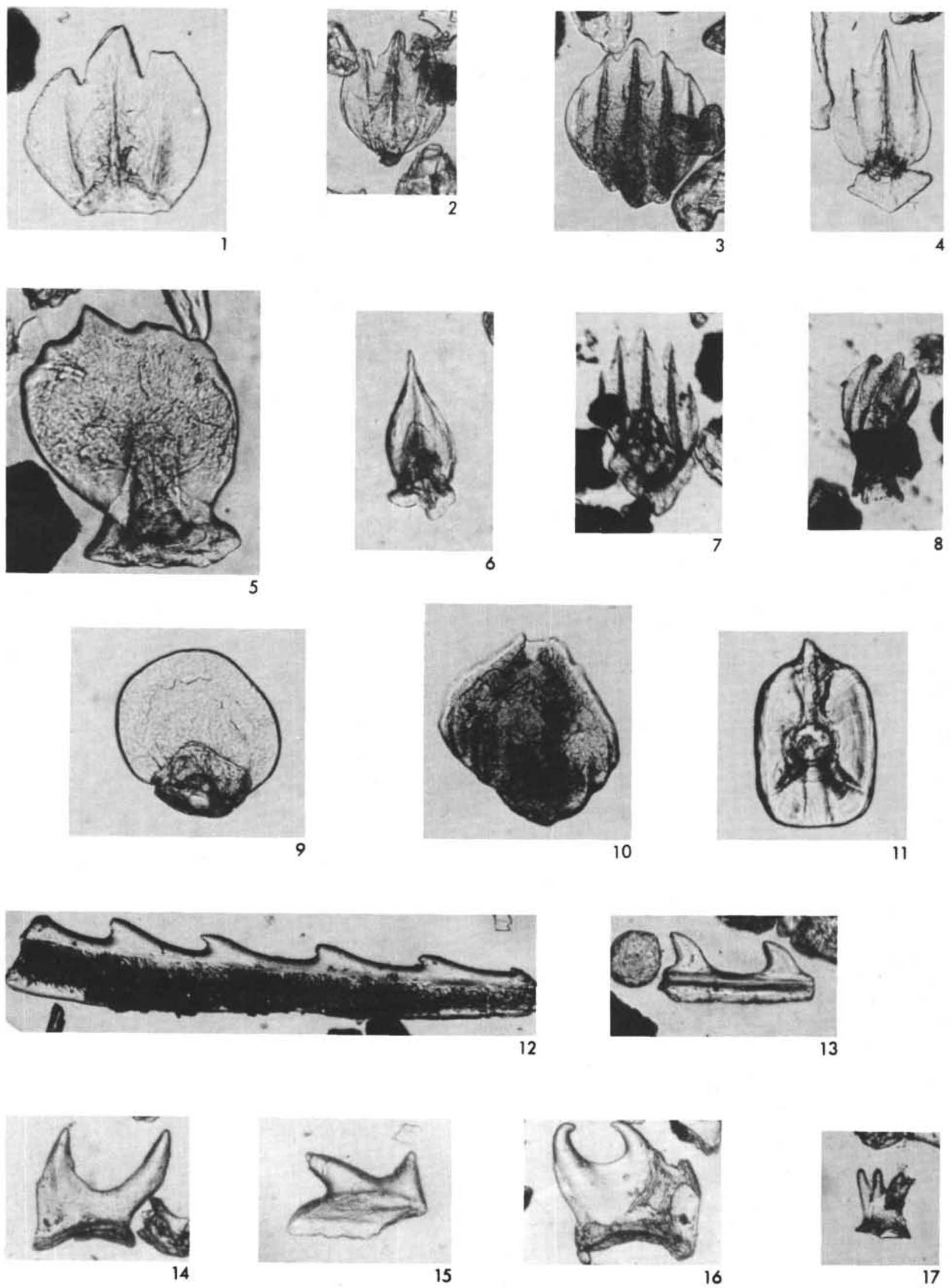
PLATE 5

(Magnification $110 \times$ )

Figures 1-10 Undescribed forms.

1. $295-3 \mathrm{~A}-1,125-129 \mathrm{~cm}, \mathrm{~S} 1.1, \mathrm{E} 22 / 0$.

2. 294-3-5, 120-124 cm, S1.3, L36/0.

3. $294-4-2,60-64 \mathrm{~cm}, \mathrm{~S} 1.2, \mathrm{R} 38 / 0$.

4. 295-3A-1, 125-129 cm, S1.1, P28/1.

5. 294-6-1, 48-52 cm, S1.4, L16/1.

6. Same sample, S1.5, H8/1.

7. 295-3A-1, 125-129 cm, S1.1, E31/0.

8. 294-4-2, 60-64 cm, S1.3, E23/0.

9. $295-3 \mathrm{~A}-1,125-129 \mathrm{~cm}, \mathrm{~S} 1.0, \mathrm{~J} 19 / 0$.

10. Same sample, S1.1, J19/0.

Figure $11 \quad a 7 / b 6 / c 3$;

Asymmetrical peak narrow depression.

294-6-1, 48-52 cm, S1.2, Q50/0.

Figures 12-17 Undescribed forms.

12. 294-3-5, 120-124 cm, S1.2, Q20/0.

13. Same sample, S1.4, F24/1.

14. 294-4-4, 65-69 cm, S1.1, Q41/4.

15. 294-3-5, 120-124 cm, S1.1, H20/0.

16. 294-3-2, 10-14 cm, S1.3, M32/0.

17. 295-3A, CC, S1.4, F6/0. 
PLATE 5
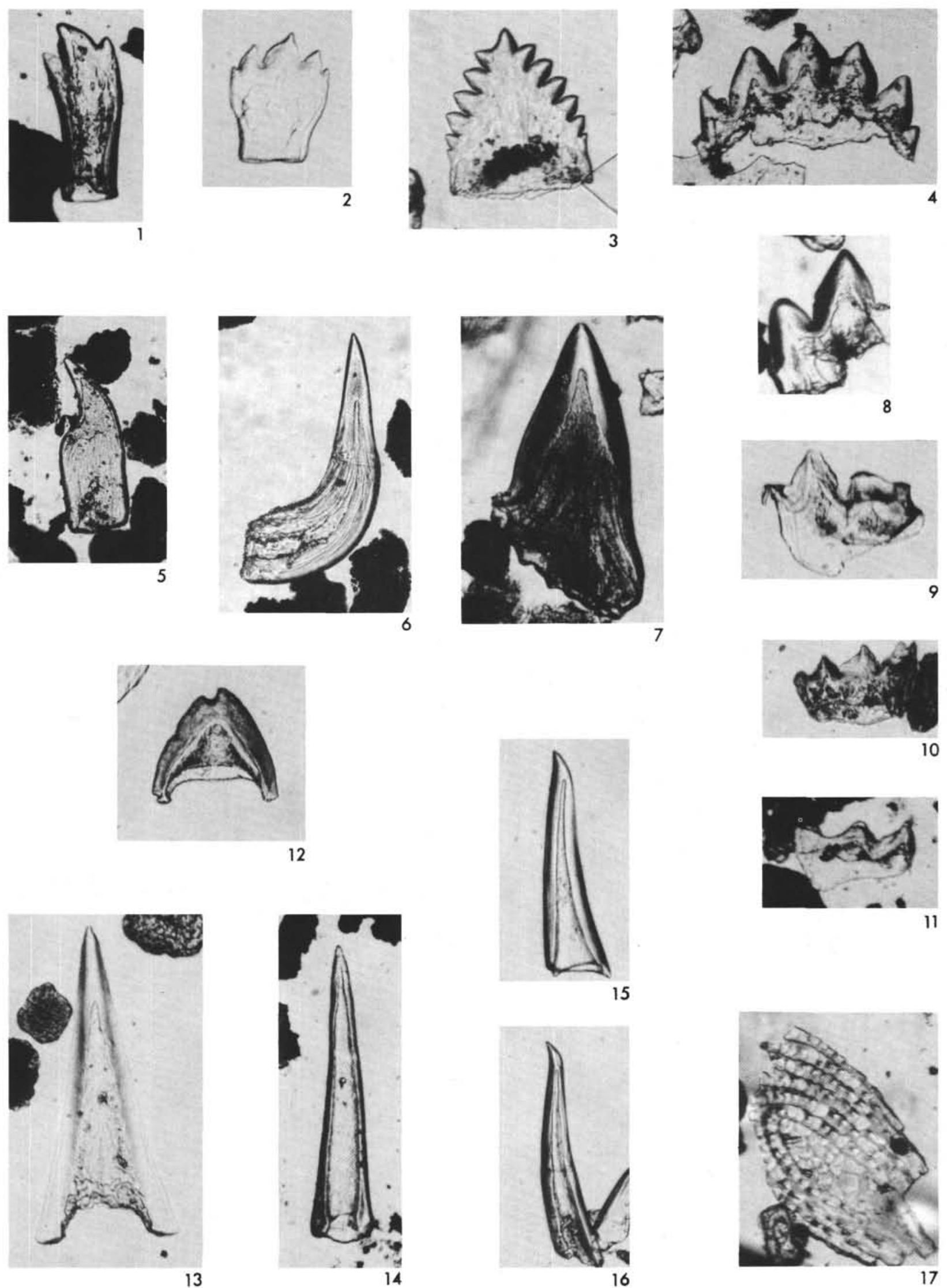